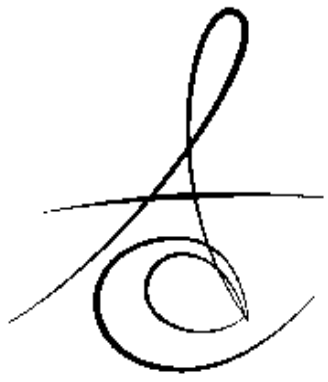

\section{MONOLİTİK ZİRKONYA SERAMİK SİSTEMLERİNİN ÜRETİM TİPLERİ İLE AŞINMA, OPTÍK VE ESTETÍK ÖZELLİKLERİ}

\section{PRODUCTION TYPES OF MONOLITHIC ZIRCONIA CERAMIC SYSTEMS WITH WEAR RESISTANCE, OPTICAL AND AESTETHIC PROPERTIES}

Doç. Dr.Rukiye DURKAN*

Makale Kodu/Article code: 2811

Makale Gönderilme tarihi: 27.04.2016

Kabul Tarihi: 22.06.2016

öz

Monolitik zirkonya seramik restorasyonlar, mükemmel mekanik özellikleri ve tek parça olarak yapılması ile veneer seramik ayrılmalarının olmadığı ve bilgisayar destekli tasarım ve bilgisayar destekli üretim (BDT/BDÜ) teknolojileri kullanılarak üretilen sistemlerdir. Bu sistemler aşınmaya karşı direnci ve estetik özellikleri ile son yıllarda birçok üretim tipi ile daha geniş kullanım alanı bulmaktadır.

Restorasyonların uzun yıllar ağızda kalabilmesi için mekanik dayanıkılığı oldukça önemlidir. Monolitik zirkonya restorasyonların yüksek aşınma dayanımı olduğu bilinmesine rağmen konvansiyonel zirkonyaya karşı üstünlükleri ve karşıt minede oluşturdukları etkiler kanıtlanamamıştır.

Opak-beyazımsı görüntüsü olan konvansiyonel zirkonya seramik sistemlere oranla yüksek translusensi gösteren monolitik zirkonyada doğal görünüm oluşturmak için sinterizasyon öncesi renklendirme yapılabilmektedir. Ayrıca yüzey parlaklığı ve estetik için parlatma ve glazür işlemleri de yapılabilmektedir. Estetik ve optik özelliklerin artırılmasında zirkonya seramik materyal tipi, translusensi özelliği ve yüzey işlemleri oldukça önemli parametrelerdir. Doğal dişler translusent olduğu için zirkonya restorasyonlarda translusensi aranan önemli bir özelliktir.

Anahtar Kelimeler: Monolitik zirkonya, estetik, aşınma direnci

\section{GİRİş}

Monolitik zirkonya seramik sistemleri, yapılan çalışmalarda kanıtlanan üstün mekanik ve optik özellikleri ile yaygın kullanım alanı bulmuştur ${ }^{1,2}$. Bu sebeple konvansiyonel zirkonya üreticileri, aynı zamanda mo-

\section{ABSTRACT}

Monolithic zirconia ceramic restorations are excellent mechanical properties in which it is not encountered veneers chipping since it is produced in one piece by using computer-aided design and computer-aided manufacturing technology (CAD /CAM). These systems with wear resistance and aesthetic properties are in widely use in recent years with many generic forms. Mechanical strength of restoration is very important to remain in the mouth for many years. Monolithic zirconia restorations could not be proved superiority against conventional zirconia and the effects they produce in the opposite enamel despite their high wear resistance known.

In order to create a natural appearance for monoblock zirconia with high translucency compared to standard zirconia ceramic systems with opaque appearance, it can be applied coloring before sintering. In addition, glazing and polishing for surface gloss and aesthetic procedures can be performed. Zirconia ceramic material type, translucency property and surface treatments are important parameters in improving the aesthetic and optical properties. The translucency of zirconia restorations is an important requisite property since natural teeth is translucent.

Keywords: Monolithic zirconia, aesthetic, wear resistance

nolitik zirkonya seramikleri piyasaya sürmüşlerdir. Günümüzde, farklı firmalara ait birçok monolitik zirkonya seramik sistemi bulunmaktadır. Bilgisayar destekli tasarım ve bilgisayar destekli üretim (BDT/BDÜ) teknolojisi ile üretilen bu sistemler ile özellikle anterior ve posterior köprüler yapılmaktadır ${ }^{2,3}$.

* Afyon Kocatepe Üniveritesi Diş Hekimliği Fakültesi, Protetik Diş Tedavisi Anabilim Dalı, Afyon

** Serbest dis hekimi, Usak

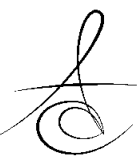


Monolitik zirkonya seramik kullanılarak yapılan resto- rasyonların aşınma özelliklerinin araştırıldığı birçok klinik çalışmada farklı sonuçlar elde edilmiştir. Karşıt diş minesinde konvansiyonel zirkonya seramik sistemlere oranla daha az, fazla ya da aynı oranda aşınma meydana geldiği belirtilmiştir ${ }^{3-6}$. Monolitik zirkonya seramiklerin konvansiyonel zirkonya seramiklere göre daha sert olan yapıları sayesinde restorasyon yüzeyinde oluşan aşınma değerlerinin daha az olduğu bulunan sonuçlar arasındadır ${ }^{6,7}$. Restorasyonda bulunan yüzey düzensizlikleri karşıt diş ve/veya restorasyonda aşınmaya neden olmaktadır. Yüzeye glazür uygulanması ile yüzey pürüzlülüğü minimalize edilir ve/veya azaltılır ${ }^{8}$. Karşıt dişlerde mine aşınmasını önlemek için parlatma işlemleri laboratuvarda özenli yapılarak ve ağızda oklüzal düzenleme yapıldıktan sonra mutlaka parlatma işlemi uygulanarak sağlanmalıdır. Zirkonya tam seramiklerde herhangi bir yüzey işlemi yapılmadan öncelikle yüzey düzeltilmeli ve sonra parlatılmadır. Aksi halde daha sonra yapılan boyama işlemlerinde ağız içinde zamanla boya materyali aşındığı zaman pürüzlü yüzey ortaya çıkar ve aşındırmayı hızlandırır ${ }^{9}$. Yüzey işlemleri, zirkonyanın aşınma değerlerine etki etmektedir ${ }^{8,10}$. Ancak monolitik zirkonya sistemlerinin abraziv etkisi hakkında fikir birliği sağlanamamıştır. Bu konuda daha fazla in vitro ve klinik sonuçlarla desteklenen in vivo çalışma yapılması gerekmektedir.

Estetik protetik restorasyonlarda materyalin renk stabilitesi ve translusensi özelliği en önemli parametrelerdir $^{11,12}$. Konvansiyonel zirkonya oldukça opak bir materyal olduğu için uygun estetik ve translusenside restorasyonlar yapabilmek amac ile porselen ile tabakalanmaktadır ${ }^{13,14}$. Konvansiyonel ve monolitik zirkonya seramik sistemleri translusensi özellikleri yönünden oldukça farklılık göstermektedir. Konvansiyonel zirkonya kor seramikler daha opak materyaller iken yeni jenerasyon ürünü olan monolitik zirkonya seramiklerin en önemli özelliklerden biri yüksek translusent özelliğe sahip olmasıdır ${ }^{11,13-16}$. Monolitik zirkonya seramiklerde, farklı yüzey işlemlerine rağmen optik ve yüzey özelliklerinin yeterli düzeyde olmadığı bildirilmektedir ${ }^{17}$. Çalışmalarda renk solüsyonları uygulamasının monolitik zirkonyada translusensi özelliğini etkilemediği belirtilmiştir ${ }^{4,17}$.

Bu derlemenin temel amacl; monolitik zirkonya seramik sistemlerinin farklı üretim tiplerini belirterek aşınma, optik ve estetik özellikleri hakkında bilgi verip, son yıllarda yapılan araştırmalardan elde edilen bilgileri sunmaktır.

Monolitik Zirkonya Seramik Sistemlerinin

\section{Üretim Tipleri}

Son yıllarda pek çok üretici firma tarafından monolitik zirkonya seramik sistemleri olan yüksek translusent zirkonya seramikler üretilmektedir. Farklı üreticilere ait monolitik zirkonya seramik sistemleri ve özellikleri Tablo 1'de gösterilmiştir ${ }^{18-26}$.

\section{Monolitik Zirkonya Seramiklerde Aşındırma Özelliği}

İdeal olarak posterior diş minesinin aşındırma etkisi molar dişlerde, yılda yaklaşık olarak 20-40 $\mu \mathrm{m}$ ve premolar dişlerde 15-18 $\mu \mathrm{m}^{\prime} \mathrm{dir}^{5}$. Buna karşın seramik restorasyonların karşıt minede oluşturduğu yıllık mine aşındırmasının 40-80 $\mu$ m olduğu belirtilmektedir ${ }^{6}$. İmplant destekli restorasyonlarda, okluzal aşınma doğal dişlere oranla 8 kat daha fazla bulunmuştur ${ }^{5}$. Monolitik zirkonya seramik restorasyonların çok sert yapıları nedeni ile özellikle karşıt diş minesinde oluşturdukları aşınma konusunda literatürde farklı yönde sonuçlar elde edilmiştir ${ }^{7}$. Farklı aşındırma simülasyon cihazları kullanılan in vitro çalışmalarda, yüksek aşınma direncine sahip olan monolitik zirkonyanın, diş minesi, sentetik hidroksiapatit ve paslamaz çelik materyalleri ile aşındırma özelliğinin benzer veya daha düşük olduğu belirtilmiştir ${ }^{6}$.

Zirkonya seramiğin, dış yüzeyleri manuel olarak ince elmas frezler ile düzeltildikten sonra polisaj işlemi kıl fırça ve elmas pasta (Dia-Glace) kullanılarak yapılır. Ayrıca elmas parlatma uçları kullanılır ${ }^{19}$. Zirkonya seramiğin yüzey düzgünlüğünden dolayı konvansiyonel seramiklere oranla benzer veya daha az mine aşındırması yaptığı belirtilmektedir ${ }^{27,28}$. Bununla birlikte zirkonya seramiğin yüzeyinde aşınma görülmemektedir $^{3,6,9,29,30}$

Monolitik zirkonya kullanılarak posterior bölgede tek tam kron yapılan 20 hastada, 6 aylık kontroller sonrasında kronlarda $10 \mu \mathrm{m}$, krona komşu olan dişte $58 \mu \mathrm{m}$, karşıt diş minesinde $112 \mu \mathrm{m}$, karşıt dişe komşu olan dişte $46 \mu \mathrm{m}$ aşınma olduğu bildirilmiştir. Monolitik zirkon yanın karşıt diş minesinde aşındırma etkisinin olduğu belirtilmiştir ${ }^{6}$.

10 hastaya yapılan monolitik zirkonya kronların, 1 yıl sonunda doğal diş minesini metal seramik kronlardan daha az ancak antagonist diş minesinden daha fazla aşındırdığı bulunmuştur ${ }^{31}$. Feldspatik seramiklerin diş minesini zirkonya seramiklere oranla daha fazla 
aşındırdığı belirtilmiştirir $27,32,33$. Ayrıca bazı çalışmalarda, monolitik zirkonya yüzeylerine yapılan düzeltme ve polisaj işlemlerinin aşındırmaya olan etkileri incelenmiştir $^{28}$. Bazı çalışmalarda, glazür yapılan zirkonya yüzeyinin aşındırııı etkisinin daha yüksek olduğu belirtilirken diğer benzer bir çalışmada, glazür yapılmayan polisaj yapılan yüzeylerin aşındırıcı etkisinin düşük olduğu rapor edilmiştir ${ }^{31,34-36}$. Son araştırmalarda glazür yapılan yüzeylerin daha parlak ve düzgün olmasına rağmen polisaj yapılan yüzeylerin daha düşük aşınmaya neden olduğu gösterilmektedir ${ }^{28,35,37-39}$. Zirkonya seramikler için özel olarak herhangi bir glazür materyali üretilmemiştir ${ }^{40}$.

Tablo 1. Monolitik zirkonya seramik sistemlerine ait özellikler

\begin{tabular}{|c|c|c|c|c|}
\hline $\begin{array}{l}\text { Seramik } \\
\text { Sistem }\end{array}$ & Üretici firma & $\begin{array}{l}\text { Sinterizasyon } \\
\text { Sıcaklığı }\left({ }^{\circ} \mathrm{C}\right)\end{array}$ & $\begin{array}{l}\text { Sinterizasyon } \\
\text { Süresi (dakika) }\end{array}$ & İçeriği \\
\hline Cercon HT & Degudent & - & - & - \\
\hline Zenostar & Wieland Dental & 1450 & 120 & $\begin{array}{l}\mathrm{ZrO}_{2}+\mathrm{HfO}_{2}+\mathrm{Y}_{2} \mathrm{O}_{3}(\%>99), \mathrm{Y}_{2} \mathrm{O}_{3}(\%>4,5), \mathrm{HfO}_{2}(\%<5) \\
\mathrm{Al}_{2} \mathrm{O}_{3}+\text { diğer oksitler }(\%<0,1) .\end{array}$ \\
\hline Prettau & Zirkonzahn & 1600 & 120 & $\begin{array}{l}\mathrm{ZrO}_{2}(\% 70-97), \mathrm{Y}_{2} \mathrm{O}_{3},(\%<4-6), \mathrm{HfO}_{2}(\%<5), \mathrm{Al}_{2} \mathrm{O}_{3}(\% \\
<1), \mathrm{SiO}_{2}(\max . \% 0,02), \mathrm{Fe}_{2} \mathrm{O}_{3}(\max . \% 0,01), \mathrm{Na}_{2} \mathrm{O} \\
\text { (max. \% 0,04). }\end{array}$ \\
\hline Ceramill Zolid & Amann Girrbach & 1450 & 120 & $\begin{array}{l}\mathrm{ZrO}_{2}+\mathrm{HfO}_{2}+\mathrm{Y}_{2} \mathrm{O}_{3}(\%>99), \mathrm{Y}_{2} \mathrm{O}_{3}(\%>4,5-5,6), \mathrm{HfO}_{2}(\% \\
<5), \mathrm{Al}_{2} \mathrm{O}_{3}(\%<0,5) \text {, Diğer oksitler }(\%<0,5) .\end{array}$ \\
\hline InCoris TZI & Sirona & 1510 & 120 & $\mathrm{ZrO}_{2}+\mathrm{HfO}_{2}+\mathrm{Y}_{2} \mathrm{O}_{3}(\% 99), \mathrm{Al}_{2} \mathrm{O}_{3}(\%<0,5), \mathrm{SiO}_{2}(\%<0,5)$. \\
\hline GC ZR Disc CIP & GC Europe & 1550 & 180 & $\begin{array}{l}\mathrm{ZrO}_{2}, \mathrm{Y}_{2} \mathrm{O}_{3}(\% 4,95-5,35), \mathrm{Al}_{2} \mathrm{O}_{3}(\% 0,15-0,35), \mathrm{SiO}_{2} \\
(\%<0,02), \mathrm{Fe}_{2} \mathrm{O}_{3}(\%<0,01), \mathrm{Na}_{2} \mathrm{O}(\%<0,04) .\end{array}$ \\
\hline $\begin{array}{l}\text { DD Bio ZM } \\
\text { translucent }\end{array}$ & Dental Direkt & 1450 & 120 & $\begin{array}{l}\mathrm{ZrO}_{2}+\mathrm{HfO}_{2}+\mathrm{Y}_{2} \mathrm{O}_{3}(\%>99), \mathrm{Al}_{2} \mathrm{O}_{3}(\%<0,25) \text {, Diğer } \\
\text { oksitler }(\%<0,25) .\end{array}$ \\
\hline $\begin{array}{l}\text { Bruxzir Solid } \\
\text { Zirconia }\end{array}$ & Glidewell & 1500 & 120 & $\begin{array}{l}\mathrm{ZrO}_{2}, \mathrm{Y}_{2} \mathrm{O}_{3}(\%<4,1), \mathrm{HfO}_{2}(\% 4), \mathrm{Al}_{2} \mathrm{O}_{3}(\% 0,34), \mathrm{SiO}_{2}( \\
\%<0,01), \mathrm{Fe}_{2} \mathrm{O}_{3}(\%<0,01), \mathrm{Na}_{2} \mathrm{O}(\%<0,01) .\end{array}$ \\
\hline Lava Plus & 3M ESPE & 1450 & 120 & - \\
\hline $\begin{array}{l}\text { Lava All } \\
\text { Zirconia }\end{array}$ & 3M ESPE & - & - & - \\
\hline NexxZr & $\begin{array}{l}\text { Sagemax } \\
\text { Bioceramic }\end{array}$ & 1500 & 120 & $\begin{array}{l}\mathrm{ZrO}_{2}+\mathrm{HfO}_{2}+\mathrm{Y}_{2} \mathrm{O}_{3}(\%>99,5), \mathrm{HfO}_{2}(\% 3), \mathrm{Al}_{2} \mathrm{O}_{3}(\%<0,15), \\
\text { Diğer oksitler }(\%<0,2) .\end{array}$ \\
\hline Katana & $\begin{array}{l}\text { Kuraray } \\
\text { Noritake Inc. }\end{array}$ & 1500 & 120 & $\begin{array}{l}\mathrm{ZrO}_{2}+\mathrm{HfO}_{2}+\mathrm{Y}_{2} \mathrm{O}_{3}(\%>99), \mathrm{Y}_{2} \mathrm{O}_{3}(\%>4,5), \mathrm{HfO}_{2}(\%<5), \\
\text { Diğer oksitler }(\%<0,1) .\end{array}$ \\
\hline ZENOTEC Zr & Ivoclar, Wieland & 1580 & 240 & - \\
\hline DiaZir & Ivoclar, Wieland & 1500 & 480 & - \\
\hline Metoxit & Liechtenstein & - & - & - \\
\hline ZirLuna & ACF Amberg & - & - & - \\
\hline ICE Zircon & Zirkonzahn & - & - & $\begin{array}{l}4-6 \% \mathrm{Y} 2 \mathrm{O} 3,<1 \% \mathrm{Al} 2 \mathrm{O} 3 \\
\max .0 .02 \% \mathrm{SiO} 2 \\
\max .0 .01 \% \mathrm{Fe} 2 \mathrm{O} 3 \\
\max .0 .04 \% \mathrm{Na} 2 \mathrm{O}\end{array}$ \\
\hline
\end{tabular}

Polisajlı zirkonya yüzeyleri karşıt diş minesinde marjinal aşınma gösterirken, zirkonya seramiğinde aşınma gözlenmemektedir. Aksine glazürlü zirkonya ve veneer uygulanmış zirkonya yüzeyleri hem karşıt diş minesinde hem de zirkonya seramiğinde aşınmaya neden olmaktadır ${ }^{30}$.

Preis ve arkadaşlarının yaptıkları in vitro çalışmada; aşınmanın pürüzlülük ve faz dönüşümü üzerine etkisinin olmadığı ancak antagonist yüzeyden madde kaybına neden olduğu sonucuna varılmıştır. Üreticinin talimatlarına göre aşınmayı önlemek ve mümkün olduğunca düşük faz dönüşümü için zirkonya yüzeyi polisajlanmalıdır ${ }^{28}$. 
Atatürk Üniv. Diş Hek. Fak. Derg.

j Dent Fac Atatürk Uni

Cilt:28, Sayı: 2, Yıl: 2018, Sayfa: 263-270
DURKAN, DESTE, ŞİMŞEK

\section{Aşınmaya Etki Eden Faktörler}

1. Seramiğin tipi ${ }^{9}$,

2. Seramiğin mikroyapısı ${ }^{9}$,

3. Seramik yüzeyinin pürüzlülüğü ${ }^{33}$,

4. Materyalin kristal ve gren büyüklüğü ${ }^{33}$,

5. Materyalin yüzey sertliği ${ }^{33}$,

6. Materyalin sürtünme dayanıklıı̆ı ${ }^{33}$,

7. Materyalin yorulma direnci ${ }^{31}$

8. Materyalin kırılma direnci ${ }^{33}$,

9. Monolitik zirkonya seramiğin yüzeyine glazür veya polisaj uygulanmass ${ }^{5}$,

10. Monolitik zirkonyanın yüzey kalitesi ${ }^{31}$,

11. Hastaya bağlı faktörler; beslenme, disfonksiyonel okluzyon, çiğneme kuvveti ve bruksizm ${ }^{5,10}$

\section{Monolitik Zirkonya Seramiklerde Renk Özelliği}

Renklendirme ve glazür işlemlerindeki gelişmeler sayesinde monolitik zirkonya restorasyonların üretimi yaygınlaşmıştır ${ }^{41,42}$. Monolitik pre-sinterize zirkonya bloklar translusent veya tabakalı olarak renkli bir şekilde üretilirler. İç renklendirme, pre-sinterize zirkonya blokların üretim aşamasında toz yapı içerisine renk vermek için metal oksitlerin ilavesi ile sağlanmaktadır $^{16,43}$. Pre-sinterize zirkonya bloklardan frezeleme yapılarak elde edilen restorasyonların sonrasında renklendirme solüsyonlarına daldırılması işlemine ise dış renklendirme denilmektedir ${ }^{43,44}$. Bunun için seryum, bizmut ve demir metal tuzlarından birisi veya kombinasyonları renklendirme solüsyonuna eklenerek renklendirme yapılmaktadır. Bu işlem, istenilen estetik etkiyi oluşturmak için değişik renk tonları oluşturulmasını mümkün kılar ${ }^{45}$.

Monolitik zirkonya ile özellikle implant üstü restorasyonlarda vertikal ve horizontal rezorbsiyona bağlı doku eksikliği durumlarında pembe boyama yapılarak dişeti estetiği sağlanmaktadır ${ }^{18}$.

Monolitik zirkonya seramik sistemlerinde, üretici firmanın önerileri doğrultusunda glazür işlemleri yapılmaktadır. Örneğin; Zenostar monolitik zirkonya seramikler için öncelikle restorasyon $1520{ }^{\circ} \mathrm{C}$ sıcaklıkta 3 saat sinterizasyon fırınında (Zenotec Fire, Wieland Dental) sinterlenir. Daha sonra restorasyonun polisaj işlemleri (Zenostar Polishing Set) ve renklendirme işlemleri (Zenostar Color Zr) yapılır. Sonrasında sprey şeklinde glazür materyali (Zenostar Magic Glaze Spray) uygulanarak $850{ }^{\circ} \mathrm{C}$ sıcaklıkta $2 \mathrm{dk}$ süre ile sinterizayonu gerçekleştirilir ${ }^{6}$.
Kim ve arkadaşlarının yaptığı çalışmada; monolitik zirkonyaya uygulanan renklendirme ile birlikte yüzey işlemlerinin; yüzey özelliklerini önemli derecede değiştirdiği, en pürüzsüz yüzeyin glazür ile elde edildiği ve bu işlemlerin translusensiye etkisinin minimal olduğu, glazürün opaklığı biraz azalttığı sonucuna varılmıştır ${ }^{42}$.

Sulaiman ve arkadaşlarının monolitik zirkonya yüzeyine gastrik asidin etkisini araştırdıkları çalışmada vardıkları sonuç; asit müdahalesi sonucunda monolitik zirkonya pürüzsüz yüzey göstermektedir ve optik özellikleri minimum etkilenmiştir. Monolitik zirkonya materyalinin pürüzsüz yüzey göstermesinin gerçek koroziv direnç olup olmadığı net bir şekilde söylenememiştir ${ }^{46}$.

Zirkonyanın yüksek opak etkisi, matriksindeki daha büyük olan partiküller nedeniyle oluşan ışık dalgası ve farklı kırınım etkisine bağlıdır. Konvansiyonel zirkonya seramikler, opak yapısından dolayı aşırı derecede renklenen destek dişlerde, metal post-kor yapılan destek dişlerde, metal dayanak kullanılan implant üstü sabit restorasyonlarda kullanılmaktadır. Konvansiyonel zirkonya seramikler, opak görünümünden dolayı özellikle estetiğin ön planda olduğu anterior bölgelerde sınırı kullanım alanına sahiptir ${ }^{13,14,47}$. Opak görünümleri ile mat ve cansız bir görüntü oluştururlar ${ }^{48}$.

Monokromatik zirkonya alt yapılar, bireysel renklendirme işlemleri, üst yapı tabakalama teknikleri ve çok renkli ve tabakalı olarak üretilen hazır blok sistemleri ile birlikte estetik sonuçlar vermektedir ${ }^{13,42}$. Ancak renk stabilitesi ile ilgili uzun süreli klinik veriler sınırlıdır ${ }^{49}$.

\section{Monolitik Zirkonya Seramiklerde Translusensi Özelliği}

Zirkonya seramiklerin translusensi özelliği, büyük oranda ışık saçıımı ile birlikte gerçekleşen ışığın bir bölümünün materyalden geçmesidir ${ }^{12}$. Bir seramiğin üzerine gelen ışığın büyük çoğunluğu yansır ve diffüz olarak dağııırsa materyal opak görünür, ışığın sadece bir kısmı dağılır, büyük çoğunluğu seramikten doğrudan geçer ise materyal translusent görünür ${ }^{12,42}$. Zirkonyanın translusensi özelliğinin tespit edilmesi farklı parametreleri içeren kompleks bir kombinasyondur $^{30,50}$

Konvansiyonel zirkonya seramik sistemlerinin translusensi düzeylerinin alümina, alümina-zirkonya ve metal alaşımlar ile benzer olduğu ifade edilmekte$\operatorname{dir}^{16,30,51}$ 
Atatürk Üniv. Diş Hek. Fak. Derg.

j Dent Fac Atatürk Uni

Cilt:28, Sayı: 2, Yıl: 2018, Sayfa: 263-270

Translusensi özelliklerini etkileyen faktörler

1. Zirkonya seramiğin kimyasal yapısı ve alümina içeriğinin miktarı ${ }^{30,50}$,

2. Seramiğin kalınlığı ${ }^{12}$,

3. Kristal yapısı ve gren büyüklüğü $16,25,30,43,50$,

4. Seramiğin fırınlanma sayısı ${ }^{4}$,

5. Veneer uygulama teknikleri ${ }^{11}$,

6. Tekrarlanan seramik boyama fırınlamalar ${ }^{11,52}$,

7. Sinterizasyon parametreleri ${ }^{30,43,50}$,

8. Uygulanan rezin simanın polimerizasyon parametreleri ${ }^{12,26}$,

9. Yüzeyin polisajlanması ${ }^{12}$,

10. Üretim aşamasındaki primer partikül boyutu ${ }^{50,52}$,

11. Materyalin içerisindeki renk pigmentleri ${ }^{53}$,

12. Materyalde görülen defekt ve pörözitelerin sayısı ve büyüklüğü $50,54,55$

13. Materyalin yüzey pürüzlülüğüü $42,56,57$.

Monolitik ve Konvansiyonel Zirkonya Seramiklerde Translusensi Özelliğinin Karşılaştırılması

Konvansiyonel zirkonya seramiklerde translusensi özelliği düşüktür. Işık geçirgenliği az olan opak materyallerdir. Konvansiyonel zirkonya kor alt yapının kalınlığı azaldıkça translusensi özelliği artmaktadır ancak direnç azalmaktadır ${ }^{58,59}$. Monolitik zirkonya seramiklerin, konvansiyonel zirkonya seramiklere oranla daha translusent olmasından dolayı kompozit rezin siman ile yapıştırılan restorasyonlarda simanın polimerizasyonu daha iyi olmaktadır ${ }^{30,50}$.

Translusensi olarak In-ceram alümina, Inceram zirkonyadan daha yüksek translusent özelliğe sahiptir. In-ceram zirkonyada lantan cam infiltrasyonu olduğu için translusensi Y-TZP'den daha yüksektir. Ancak her iki materyalin de posterior bölgede, estetiğin önemli olmadığı ve opaklığın gerekli olduğu vakalarda kullanılması önerilmektedir ${ }^{60}$. Lava zirkonya korun, 0,3 ve $0,5 \mathrm{~mm}$ kalınlıkta yüksek translusent olduğu belirtilmiştir ${ }^{55,56,60}$. Zirkonyanın, cam seramiklere oranla translusensliği daha düşüktür, ancak zirkonya kalınlığa bağlı translusent değişikliğine daha az hassastır ${ }^{30}$.

Matsuzaki ve arkadaşlarının yaptığı çalışmada vardığı sonuç; farklı renklerde monolitik zirkonya konvansiyonel opak zirkonyaya göre daha yüksek translusensi göstermektedir ve veneer porselen içermemesine rağmen porselene benzer renk değerleri vermektedir. Translusent zirkonya, opak zirkonya ile benzer seviyede dayanıklılık göstermektedir ${ }^{14}$.
DURKAN, DESTE, ŞİMŞEK

\section{SONUÇ}

Monolitik zirkonya seramik sistemlerinin günümüzde kullanımının yaygınlaşması ile birlikte birçok konvansiyonel zirkonya üreticisi monolitik zirkonya üretimine başlamıştır. Yapılan çalışmalarda monolitik zirkonyanın estetik ve optik özellikleri değerlendirilmiş ve posterior restorasyonlara ek olarak estetik bölgede de kullanılabilecek bir materyal olduğu bildirilmiştir. Monolitik zirkonyanın aşınma dayanımı ve karşıt diş minesinde oluşturduğu aşınma konusunda literatürde fikir birliği mevcut değildir. Bu konuda daha fazla çalışma yapılması gerekmektedir.

Monolitik zirkonya seramik sistemleri ve bilgisayar destekli restorasyon üretim teknolojileri bugün pek çok yeni gelişmeye imkan sağlayarak ve yaygınlaşarak kullanımı artmaktadır. Gelecek dönemlerde materyal üretimi özelliklerinin idealize edilmesi ile hasta ve hekimlerin yüksek oranda kullanacağı restorasyonlar yapılacaktır. Özellikle nano boyutlarda biyoteknolojik üretimler ile protetik tedavilerde çok önemli gelişmeler olacaktır

Rukiye Durkan, ORCID ID: 0000-0002-3381-4073 Gonca Deste, ORCID ID: 0000-0002-5481-0063

\section{KAYNAKLAR}

1. Nakamura $K$, Harada $A$, Inagaki $R$, Kanno $T$, Niwano $Y$, Milleding $P$, Örtengren $U$. Fracture resistance of monolithic zirconia molar crowns with reduced thickness. Acta Odontol Scand 2015;73:602-8.

2. Beuer F, Edelhoff D, Gernet W, Naumann M. Effect of preparation angles on the precision of zirconia crown copings fabricated by CAD/CAM system. Dent Mater J 2008;27:814-20.

3. Preis V, Behr M, Kolbeck C, Hahnel S, Handel G, Rosentritt $M$. Wear performance of substructure ceramics and veneering porcelains. Dent Mater 2011;27:796-804.

4. Kim HK, Kim SH. Effect of the number of coloring liquid applications on the optical properties of monolithic zirconia. Dent Mater 2014;30:229-37.

5. Cardelli P, Manobianco FP, Serafini N, Murmura G, Beuer F. Full-arch, implant-supported monolithic zirconia rehabilitations: pilot clinical evaluation of wear against natural or composite teeth. J Prosthet Dent 2015;10.1111:1-5. 
6. Stober T, Bermejo JL, Rammelsberg P, Schmitter M. Enamel wear caused by monolithic zirconia crowns after 6 months of clinical use. J Oral Rehabil 2014;41:314-22.

7. Preis V, Weiser F, Handel G, Rosentritt M. Wear performance of monolithic dental ceramics with different surface treatments. Quintessence Int 2013;44:393-405.

8. Bindl $A$, Lüthy $H$, Mörmann WH. Strength and fracture pattern of monolithic CAD/CAM-generated posterior crowns. Dent Mater 2006;22:29-36.

9. Park JH, Park S, Lee $K$, Yun KD, Lim HP. Antagonist wear of three CAD/CAM anatomic contour zirconia ceramics. J Prosthet Dent 2014;111:20-9.

10. Oh WS, Delong R, Anusavice KJ. Factors affecting enamel and ceramic wear: a literature review. J Prosthet Dent 2002;87:451-9.

11. Harianawala HH, Kheur, MG, Apte SK, Kale BB, Sethı TS, Kheur SM. Comperative analyse of transmittance for different types of commercially avaible zirconia and lithium disilicate materials. ] Adv Prosthodont 2014;6:456-61.

12. Sulaiman TA, Abdulmajeed AA, Donovan TE, Ritter AV, Vallittu PK, Närhi TO, Lassila LV. Optical properties and light irradiance of monolithic zirconia at variable thicknesses. Dent Mater 2015;31:1180-7.

13. Ueda K, Güth JF, Erdelt K, Stimmelmayr M, Kappert $\mathrm{H}$, Beuer $\mathrm{F}$. Light transmittance by a multicoloured zirconia material. Dent Mater J 2015;34:310-4.

14. Matsuzaki F, Sekine $H$, Honma S, Takanashi T, Furuya K, Yajima Y, Yoshinari M. Translucency and flexural strength of monolithic translucent zirconia and porcelain-layered zirconia. Dent Mater J 2015;34:910-7.

15. Nordahl N, Vult von Steyern P, Larsson C. Fracture strength of ceramic monolithic crown systems of different thickness. J Oral Sci 2015;57:255-61.

16. Zhang F, Vanmeensel K, Batuk M, Hadermann J, Inokoshi M, Van Meerbeek B, Naert I, Vleugels J. Highly-translucent, strong and aging-resistant 3Y-TZP ceramics for dental restoration by grain boundary segregation. Acta Biomater 2015;16:215-22.
17. Kim HK, Kim SH, Lee JB, Han JS. Effect of polishing and glazing on the color and spectral distribution of monolithic zirconia. J Adv Prosthodont 2013;5:296-304.

18. Sadid-Zadeh R, Liu PR, Aponte-Wesson R, O'neal SJ. Maxillary cement retained implant supported monolithic zirconia prosthesis in a full mouth rehabilitation: a clinical report. J Adv Prosthodont 2013;5:209-17.

19. Stawarczyk B, Ozcan M, Hallmann L, Ender A, Mehl A, Hammerlet $\mathrm{CH}$. The effect of zirconia sintering temperature on flexural strength, grain size, and contrast ratio. Clin Oral Investig 2013a;17:269-74.

20. Bavbek NC, Roulet JF, Ozcan M. Evaluation of microshear bond strength of orthodontic resin cement to monolithic zirconium oxide as a function of surface conditioning method. J Adhes Dent 2014;16:473-80.

21. Majić Renjo M, Curkovic L, Stefancıc S, Corıc D. Indentation size effect of Y-TZP dental ceramics. Dent Mater 2014;30:371-6.

22. Sripetchdanond J, Leevailoj C. Wear of human enamel opposing monolithic zirconia, glass ceramic, and composite resin: an in vitro study. J Prosthet Dent 2014;112:1141-50.

23. Sun T, Zhou S, Lai R, Liu R, Ma S, Zhou Z, Longquan $S$. Load-bearing capacity and the recommended thickness of dental monolithic zirconia single crowns. J Mech Behav Biomed Mater 2014;35:93-101.

24. Zesewitz TF, Knauber AW, Northdurft FP. Fracture resistance of a selection of full-contour all-ceramic crowns: an in vitro study. Int J Prosthodont 2014;27:264-6.

25. Qeblawi DM, Campillo-Funollet M, Muñoz CA. In vitro shear bond strength of two self-adhesive resin cements to zirconia. J Prosthet Dent 2015;113:122-7.

26. Sulaiman TA, Abdulmajeed AA, Donovan TE, Ritter $A V$, Lassila LV, Vallittu PK, Närhi TO. Degree of conversion of dual-polymerizing cements light polymerized through monolithic zirconia of different thicknesses and types. J Prosthet Dent 2015;114:103-8.

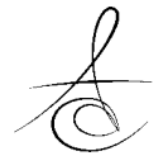


27. Venezia $P$, Torsello $F$, Cavalcanti R, D'Amato $S$. Retrospective analysis of 26 complete-arch implant-supported monolithic zirconia prostheses with feldspathic porcelain veneering limited to the facial surface. J Prosthet Dent 2015;114:506-12.

28. Preis V, Schmalzbauer M, Bougeard D, SchneiderFeyrer S, Rosentritt M. Surface properties of monolithic zirconia after dental adjustment treatments and in vitro wear simulation. J Dent 2015;43:133-9.

29. Jung YS, Lee JW, Choi YJ, Ahn JS, Shin SW, Huh JB. A study on the in-vitro wear of the natural tooth structure by opposing zirconia or dental porcelain. J Adv Prosthodont 2010;2:111-5.

30. Stawarczyk B, Frevert K, Ender A, Roos M, Sener $B$, Wimmer $T$. Comparison of four monolithic zirconia materials with conventional ones: Contrast ratio, grain size, four-point flexural strength and two-body wear. J Mech Behav Biomed Mater 2015;59:128-38.

31. Mundhe K, Jain V, Pruthi G, Shah N. Clinical study to evaluate the wear of natural enamel antagonist to zirconia and metal ceramic crowns. J Prosthet Dent 2015;114:358-63.

32. Cheng $\mathrm{CW}$, Chien $\mathrm{CH}$, Chen $\mathrm{CJ}$, Papaspyridakos, $\mathrm{P}$. Complete-mouth implant rehabilitation with modified monolithic zirconia implant-supported fixed dental prostheses and an immediate-loading protocol: a clinical report. J Prosthet Dent 2013;109:347-52.

33. Amer R, Kürklü $D$, Johnston W. Effect of simulated mastication on the surface roughness of three ceramic systems. J Prosthet Dent 2015;114:260-5.

34. Cattani-Lorente $M$, Durual $S$, Amez-Droz M, Wiskott HW, Scherrer SS. Hydrothermal degradation of a 3Y-TZP translucent dental ceramic: A comparison of numerical predictions with experimental data after 2 years of aging. 2016;32:394-402.

35. Janyavula S, Lawson N, Cakir D, Beck P, Ramp LC, Burgess JO. The wear of polished and glazed zirconia against enamel. J Prosthet Dent 2013;109:22-9.

36. Amer R, Kurklu D, Kateeb E, Seghi RR. Three body wear potential of dental yttrium-stabilized zirconia ceramic after grinding, polishing, and glazing treatments. J Prosthet Dent 2014;112:1151-5.
37. Mitov G, Heintze SD, Walz S, Woll K, Muecklıch F, Pospiech P. Wear behavior of dental Y-TZP ceramic against natural enamel after different finishing procedures. Dent Mater 2012;28:909-18.

38. Kontos L, Schille C, Schweizer E, Geis-Gerstorfer J. Influence of surface treatment on the wear of solid zirconia. Acta Odontol Scand 2013;71:482-7.

39. Sabrah AH, Cook NB, Luangruangrong P, Hara AT, Bottino MC. Full-contour Y-TZP ceramic surface roughness effect on synthetic hydroxyapatite wear. Dent Mater 2013;29:666-73.

40. Johansson C, Kmet G, Rivera J, Larsson C, Vult Von Steyern $P$. Fracture strength of monolithic allceramic crowns made of high translucent yttrium oxide-stabilized zirconium dioxide compared to porcelain-veneered crowns and lithium disilicate crowns. Acta Odontol Scand 2014;72:145-153.

41. Ha SR. Biomechanical three-dimensional finite element analysis of monolithic zirconia crown with different cement type. J Adv Prosthodont 2015;7:475-83.

42. Kim HK, Kim SH, Lee JB, Ha SR. Effects of surface treatments on the translucency, opalescence, and surface texture of dental monolithic zirconia ceramics. J Prosthet Dent 2016;115;773-9.

43. Sulaiman $\quad T A$, Abdulmajeed $\quad A A$, Donovan TE, Vallittu PK, Närhi TO, Lassila LV. The effect of staining and vacuum sintering on optical and mechanical properties of partially and fully stabilized monolithic zirconia. Dent Mater J 2015;34:605-10.

44. Kurtulmus-Yılmaz S, Ulusoy M. Comparison of the translucency of shaded zirconia all-ceramic systems. J Adv Prosthodont 2014;6:415-22.

45. Denry I, Kelly JR. State of the art o zirconia for dental applications. Dent Mater 2008;24:299-307.

46. Sulaiman TA, Abdulmajeed AA, Shahramian K, Hupa L, Donovan TE, Vallittu P, Närhi TO. Impact of gastric acidic challenge on surface topography and optical properties of monolithic zirconia. Dent Mater 2015;31:1445-52.

47. Srikanth R, Kosmac T, Della Bona A, Yin L, Zhang $Y$. Effects of cementation surface modifications on fracture resistance of zirconia. Dent Mater 2015;31:435-42. 
48. Heffernan MJ, Aquilino SA, Diaz-Arnold, AM, Haselton DR, Stanford, CM, Vargas MA. Relative translucency of six all-ceramic systems. Part I: core materials. J Prosthet Dent 2002;88:4-9.

49. Herrguth $M$, Wichmann $M$, Reich $S$. The aesthetics of all-ceramic venered and monolithic CAD/CAM crowns. J Oral Rehabil 2005;32:747-52.

50. Ilie N, Stawarczyk B. Quantification of the amount of blue light passing through monolithic zirconia with respect to thickness and polymerization conditions. J Prosthet Dent 2015;113:114-21.

51. Heffernan MJ, Aquilino SA, Diaz-Arnold, AM, Haselton DR, Stanford, CM, Vargas MA. Relative translucency of six all-ceramic systems. Part II: core and veneer materials. J Prosthet Dent 2002;88:10-5.

52. Klimke J, Trunec M, Krell A. Transparent tetragonal yttria-stabilized zirconia ceramics: influence of scattering caused by birefringence. J Am Ceram Soc 2011;94:1850-8.

53. Ebeid K, Wille S, Hamdy A, Salah T, El-Etreby A, Kern $M$. Effect of changes in sintering parameters on monolithic translucent zirconia. Dent Mater 2014;30:419-24.

54. Yu Zhang. Making yttria-stabilized tetragonal zirconia translucent. Dent Mater 2014;30:1195203.

55. Heather JC, Seong WJ, Peson IJ. Current ceramic materials and systems with clinical recommendations: A systematic review. J Prosthet Dent 2007;98:389-404.

56. Vichi A, Louca C, Corciolani G, Ferrari M. Color related to ceramic and zirconia restorations: a review. Dent Mater 2011;27:97-108.

57. Akar GC, Pekkan G, Cal E, Eskitaşçıŏlu G, Ozcan $M$. Effects of surface-finishing protocols on the roughness, color change, and translucency of different ceramic systems. J Prosthet Dent 2014;112:314-21.

58. Wang F, Takahashi $H$, Iwasaki N. Translucency of dental ceramics with different thicknesses. J Prosthet Dent 2013;110:14-20.

59. Sinmazisik G, Tarcın B, Demirbas B, Gulmez T, Bor $E$, Ozer $F$. The effect of zirconia thickness on the biaxial flexural strenght of zirconia ceramic bilayered discs. Dent Mater J 2015;34:640-7.
60. Bayramoğlu E, Özkan Y. Comparing of glass ceramic restorations and zirconia based ceramic restorations. J Dent Fac Atatürk Uni Supplement. 2012;5:110-123.

\author{
Yazışma Adresi \\ Doç. Dr.Rukiye DURKAN \\ AKÜ Diş Hekimliği Fakültesi \\ Protetik Diş Tedavisi Anabilim Dalı \\ TIf: 05304152496 \\ e-mail: rukiye_durkan@hotmail.com
}

